\title{
Oxygen Atom Transfer from Pyridylpyridazine Chelated Oxidorhenium(V) Complex to Diphosphines: Twin Isomerization, Effects of Diphosphine Spacer Length and Metal Oxidation State
}

\author{
BIKASH KUMAR PANDA ${ }^{1}$, BISWARUP MONDAL ${ }^{2}$, \\ UMASANKAR SENAPATI $^{3}$ and SUMAN SENGUPTA ${ }^{4 *}$
}

${ }^{1}$ Department of Chemistry, Jangipur College, Jangipur, Murshidabad - 742213, W.B., India

${ }^{2}$ Department of Chemistry, Sitananda College, Nandigram, Purba Medinipur-721631, W.B., India

${ }^{3}$ Department of Chemistry, Santipur College, Santipur, Nadia-741404, W.B., India

${ }^{4}$ Department of Chemistry, Ananda Chandra College, Jalpaiguri-735101, W.B., India

sengupta.sum@gmail.com

Received 3 March 2015 / Accepted 18 March 2015

\begin{abstract}
The bimolecular reaction of $\mathrm{Re}^{\mathrm{V}} \mathrm{OCl}_{3}(\mathrm{dppz}), \mathbf{1}$, (where dppz is 3,6-di(2-pyridyl) pyridazine) with excess diphosphine $\mathrm{Ph}_{2} \mathrm{P}\left(\mathrm{CH}_{2}\right)_{\mathrm{x}} \mathrm{PPh}_{2}(\mathrm{x}=1-4)$ has furnished $\mathrm{Re}^{\mathrm{III}}\left(\mathrm{OPPh}_{2}\left(\mathrm{CH}_{2}\right)_{\mathrm{x}}\right.$ $\left.\mathrm{PPh}_{2}\right) \mathrm{Cl}_{3}(\mathrm{dppz}), 2$ with a dangling phosphine function which is spontaneously converted in solution to $\mathrm{Re}^{\mathrm{III}}\left(\mathrm{PPh}_{2}\left(\mathrm{CH}_{2}\right)_{\mathrm{x}} \mathrm{P}(\mathrm{O}) \mathrm{Ph}_{2}\right) \mathrm{Cl}_{3}(\mathrm{dppz})$, 3. The complex $2(\mathrm{x}=1)$ on reaction with excess 1 affords mononuclear $\mathrm{Re}^{\mathrm{III}}\left(\mathrm{OPPh}_{2}\left(\mathrm{CH}_{2}\right) \mathrm{P}(\mathrm{O}) \mathrm{Ph}_{2}\right) \mathrm{Cl}_{3}(\mathrm{dppz}), 4$ while for $\mathrm{x}=2-4$ binuclear complex (dppz)Cl $\mathrm{Re}^{\mathrm{III}}\left(\mathrm{OPPh}_{2}\left(\mathrm{CH}_{2}\right)_{\mathrm{x}} \mathrm{PPh}_{2} \mathrm{O}\right) \mathrm{Re}^{\mathrm{III}} \mathrm{Cl}_{3}(\mathrm{dppz}), 5$ was obtained. Rate studies of the twin isomerization (linkage-cum-geometrical) reaction $2 \rightarrow 3$ revealed that the reaction is intramolecular in nature. It is initiated by the nucleophilic attack of the metal by the dangling phosphine function. The process slows down nearly exponentially as the diphosphine spacer length $x$ increases. The oxidized complex $\left[\mathrm{Re}^{\mathrm{IV}}\left(\mathrm{OPPh}_{2}\left(\mathrm{CH}_{2}\right) \mathrm{PPh}_{2}\right) \mathrm{Cl}_{3}(\mathrm{dppz})\right] \mathrm{ReO}_{4}, 6$ does not isomerize.
\end{abstract}

Keywords: Oxygen atom transfer, Twin isomerization, Pyridylpyridazine, Rhenium

\section{Introduction}

Different groups of oxidorhenium(V) complexes have attracted considerable interest for three reasons. First, they find extensive application because ${ }^{186} \mathrm{Re}\left(90 \mathrm{~h} t_{1 / 2}, 1.02 \mathrm{MeV} \beta\right.$, $137 \mathrm{keV} \chi(7 \%))$ and ${ }^{188} \operatorname{Re}\left(17 \mathrm{ht} t_{1 / 2}, 2.11 \mathrm{MeV} \beta, 155 \mathrm{keV} \chi(15 \%)\right)$ emit $\beta^{-}$particles of therapeutically useful energies ${ }^{1-3}$. Second, the rhenium complexes are structural models of 
their less easily handled technetium $(99 \mathrm{mTc})$ analogues that find use in nuclear medicine, particularly as heart imaging agents ${ }^{1}$. Third, many such rhenium compounds catalyze oxygen atom transfer (OAT) reactions, as recently reviewed ${ }^{3-5}$. Our interest in the Re(III) complexes stems from the expectation that $\mathrm{d}^{4} \mathrm{Re}(\mathrm{III})$ complexes will be kinetically more inert than $\mathrm{d}^{2} \operatorname{Re}(\mathrm{V})$ complexes and, thus, may be useful for radio therapeutic applications ${ }^{1-5}$. Recently we reported oxido chelate $\mathrm{Re}^{\mathrm{V}} \mathrm{OCl}_{3}(\mathrm{dppz})$, 1, where dppz is 3,6-di(2-pyridyl) pyridazine) which underwent facile bimolecular oxygen atom transfer reaction with triphenyl phosphine furnishing the corresponding phosphine oxide complex $\mathrm{Re}^{\mathrm{III}}\left(\mathrm{OPPh}_{3}\right) \mathrm{Cl}_{3}(\mathrm{dppz})$ as shown in Eq. $1^{6}$.

$$
\mathrm{ReOCl}_{3}(\mathrm{dppz})+\mathrm{PPh}_{3} \rightarrow \mathrm{Re}\left(\mathrm{OPPh}_{3}\right) \mathrm{Cl}_{3}(\mathrm{dppz})
$$

The present work was initiated to scrutinize the transfer reactions of $\mathbf{1}$ with a group of linear diphosphines bearing a polymethylene spacer of variable length. Attention has been focused on the transfer of one oxygen atom only so that phosphine oxide complexes $\mathrm{Re}^{\mathrm{III}}\left(\mathrm{OPPh}_{2}\left(\mathrm{CH}_{2}\right)_{\mathrm{x}} \mathrm{PPh}_{2}\right) \mathrm{Cl}_{3}(\mathrm{dppz}), 2(\mathrm{x}=1-4)$ with a dangling phosphine function are generated.

The latter function could be a potential nucleophile that might attack the metal site within the same molecule. Such reactivity has indeed been observed providing an opportunity to scrutinize the effects of diphosphine spacer length and metal oxidation state. Oxygen atom transfer to the pendant phosphine function of $\mathbf{2}$ occurs upon reacting it with excess of 1. To model the observed phenomenon the results of monophosphorus species of type $\mathrm{Re}^{\mathrm{III}}\left(\mathrm{OPPh}_{3}\right), \mathrm{Re}^{\mathrm{IV}}\left(\mathrm{OPPh}_{3}\right)$ and $\mathrm{Re}^{\mathrm{III}}\left(\mathrm{PPh}_{3}\right)$ have been compared and the factors controlling the reactivity are scrutinized.

\section{Experimental}

Four diphosphines $\mathrm{Ph}_{2} \mathrm{P}\left(\mathrm{CH}_{2}\right)_{\mathrm{x}} \mathrm{PPh}_{2}(\mathrm{x}=1-4)$ have been employed in the present work. In order to achieve transfer of only one oxygen atom to the diphosphines, excess phosphine was reacted (Eq. 2) with 1 in dichloromethane solution furnishing the species of type $\mathrm{Re}^{\mathrm{III}}\left(\mathrm{OPPh}_{2}\left(\mathrm{CH}_{2}\right)_{\mathrm{X}} \mathrm{PPh}_{2}\right) \mathrm{Cl}_{3}(\mathrm{dppz}), 2$. The rate of the reaction of Eq. 2 has been determined spectrophotometrically in dichloromethane solution at $308 \mathrm{~K}$ for the $\mathrm{x}=4$ case where the subsequent isomerization proceeds very slowly. In the presence of excess diphosphine the rate of transfer is proportional to the concentration of $\mathbf{1}$ and the observed rate constant is proportional to the concentration of phosphine implying a second order rate law.

$$
\mathrm{ReOCl}_{3}(\mathrm{dppz})+\mathrm{PPh}_{2}\left(\mathrm{CH}_{2}\right)_{\mathrm{x}} \mathrm{PPh}_{2} \rightarrow \mathrm{Re}\left(\mathrm{OPPh}_{2}\left(\mathrm{CH}_{2}\right)_{\mathrm{x}} \mathrm{PPh}_{2}\right) \mathrm{Cl}_{3}(\mathrm{dppz})
$$

The complexes of type $\mathbf{2}$ are inherently reactive in dichloromethane solution, the dangling phosphine function displacing the coordinated phosphine oxide function furnishing $\operatorname{Re}^{\mathrm{III}}\left(\mathrm{PPh}_{2}\left(\mathrm{CH}_{2}\right)_{\mathrm{x}} \mathrm{P}(\mathrm{O}) \mathrm{Ph}_{2}\right) \mathrm{Cl}_{3}(\mathrm{dppz}), 3$ (Eq. 3). This linkage isomerization is simultaneously attended by meridional $\rightarrow$ facial geometrical isomerization; the net result is twin isomerization.<smiles></smiles>

$\operatorname{ReOCl}_{3}(\mathrm{dppz}), 1$

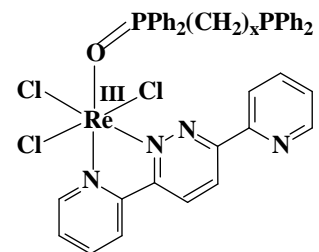

$\operatorname{Re}\left(\mathrm{OPPh}_{2}\left(\mathrm{CH}_{2}\right)_{\mathbf{X P h}} \mathrm{PPCl}_{3}(\mathrm{dppz}), 2\right.$

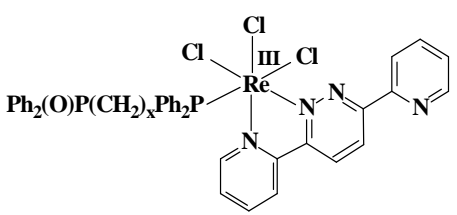

$\operatorname{Re}\left(\mathrm{PPh}_{2}\left(\mathrm{CH}_{2}\right)_{\mathrm{x}} \mathrm{P}(\mathrm{O}) \mathrm{Ph}_{2}\right) \mathrm{Cl}_{3}(\mathrm{dppz}), 3$ 


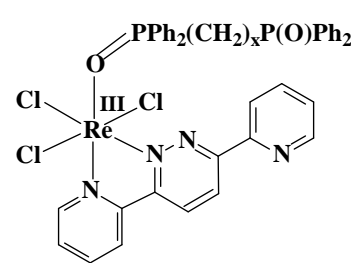

$\operatorname{Re}\left(\mathrm{OPPh}_{2}\left(\mathrm{CH}_{2}\right)_{\mathrm{X}} \mathrm{P}(\mathrm{O}) \mathrm{Ph}_{2}\right) \mathrm{Cl}_{3}(\mathrm{dppz}), 4$

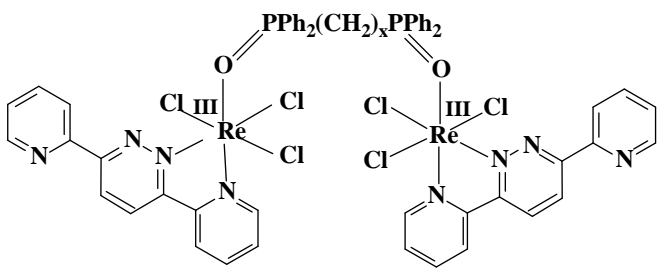

$(\mathrm{dppz}) \mathrm{Cl}_{3} \operatorname{Re}\left(\mathrm{OPPh}_{2}\left(\mathrm{CH}_{2}\right)_{\mathrm{x}} \mathrm{PPh}_{2} \mathrm{O}\right) \mathrm{ReCl}_{3}(\mathrm{dppz}),(\mathrm{x}=2-4), 5$

$$
\mathrm{Re}\left(\mathrm{OPPh}_{2}\left(\mathrm{CH}_{2}\right)_{\mathrm{x}} \mathrm{PPh}_{2}\right) \mathrm{Cl}_{3}(\mathrm{dppz}) \rightarrow \mathrm{Re}^{\mathrm{III}}\left(\mathrm{PPh}_{2}\left(\mathrm{CH}_{2}\right)_{\mathrm{x}} \mathrm{P}(\mathrm{O}) \mathrm{Ph}_{2}\right) \mathrm{Cl}_{3}(\mathrm{dppz})
$$

Oxygen atom transfer to the pendant phosphine function of $\mathbf{2}$ occurs upon reacting it with excess of $\mathbf{1}$. For $\mathrm{x}=1$ only mononuclear product $\mathrm{Re}^{\mathrm{III}}\left(\mathrm{OPPh}_{2}\left(\mathrm{CH}_{2}\right) \mathrm{P}(\mathrm{O}) \mathrm{Ph}_{2}\right) \mathrm{Cl}_{3}(\mathrm{dppz})$, 4 (Eq. 4) incorporating a dangling phosphine oxide function could be isolated. No binuclear product was observable. For $\mathrm{x}=2-4$ binuclear complex $(\mathrm{dppz}) \mathrm{Cl}_{3} \mathrm{Re}^{\mathrm{III}}\left(\mathrm{OPPh}_{2}\left(\mathrm{CH}_{2}\right)_{\mathrm{x}}\right.$ $\mathrm{PPh}_{2} \mathrm{O}$ ) $\mathrm{Re}^{\mathrm{III}} \mathrm{Cl}_{3}$ (dppz), 5, (Eq. 5), insoluble in all common organic solvents is obtained. Its composition and gross connectivity as in $\mathbf{5}$ is however not subject to any serious question. ${ }^{7,8}$

$$
\begin{aligned}
& \operatorname{Re}\left(\mathrm{OPPh}_{2}\left(\mathrm{CH}_{2}\right) \mathrm{PPh}_{2}\right) \mathrm{Cl}_{3}(\mathrm{dppz})+\mathrm{ReOCl}_{3}(\mathrm{dppz}) \rightarrow \mathrm{Re}^{\mathrm{III}}\left(\mathrm{OPPh}_{2}\left(\mathrm{CH}_{2}\right) \mathrm{PPh}_{2} \mathrm{O}\right) \mathrm{Cl}_{3}(\mathrm{dppz}) \\
& \operatorname{Re}\left(\mathrm{OPPh}_{2}\left(\mathrm{CH}_{2}\right)_{\mathrm{x}} \mathrm{PPh}_{2}\right) \mathrm{Cl}_{3}(\mathrm{dppz})+\mathrm{ReOCl}_{3}(\mathrm{dppz}) \rightarrow \\
& (\mathrm{dppz}) \mathrm{Cl}_{3} \mathrm{Re}^{\mathrm{III}}\left(\mathrm{OPPh}_{2}\left(\mathrm{CH}_{2}\right)_{\mathrm{x}} \mathrm{PPh}_{2} \mathrm{O}\right) \mathrm{Re}^{\mathrm{III}} \mathrm{Cl}_{3}(\mathrm{dppz})
\end{aligned}
$$

The phosphine oxide complex $2(\mathrm{x}=1)$ underwent facile metal oxidation upon treatment with dilute nitric acid in aceonitrile solution furnishing $1: 1$ electrolytic $\left(\Lambda, 97 \Omega^{-1} \mathrm{~cm}^{2} \mathrm{~mol}^{-1}\right.$ in methanol solution) $\left[\mathrm{Re}^{\mathrm{IV}}\left(\mathrm{OPPh}_{2}\left(\mathrm{CH}_{2}\right) \mathrm{PPh}_{2}\right) \mathrm{Cl}_{3}(\mathrm{dppz})\right] \mathrm{ReO}_{4}, 6$ (Eq. 6) as yellow solid.

$$
\mathrm{Re}\left(\mathrm{OPPh}_{2}\left(\mathrm{CH}_{2}\right) \mathrm{PPh}_{2}\right) \mathrm{Cl}_{3}(\mathrm{dppz}) \rightarrow\left[\mathrm{Re}^{\mathrm{IV}}\left(\mathrm{OPPh}_{2}\left(\mathrm{CH}_{2}\right) \mathrm{PPh}_{2}\right) \mathrm{Cl}_{3}(\mathrm{dppz})\right] \mathrm{ReO}_{4}
$$

It is unclear how the $\mathrm{ReO}_{4}^{-}$anion originated from the starting material but this has precedence in rhenium chemistry ${ }^{6,8,9}$.

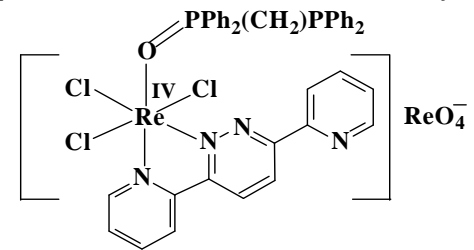

$\left[\operatorname{Re}\left(\mathrm{OPPh}_{2}\left(\mathrm{CH}_{2}\right) \mathrm{PPh}_{2}\right) \mathrm{Cl}_{3}(\mathrm{dppz})\right] \mathrm{ReO}_{4}, 6$

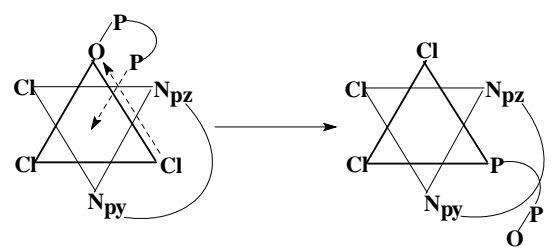

7

\section{Materials}

The $\mathrm{ReOCl}_{3}(\mathrm{dppz})$ complexes were prepared by literature method ${ }^{6}$. HPLC grade acetonitrile was used for electrochemical work and all other chemicals and solvents were of reagent grade and were used as received.

\section{Physical measurements}

UV-Vis spectral measurements were carried out with a Shimadzu UVPC 1601 spectrometer fitted with thermostated cell compartments. IR spectra $\left(4000-100 \mathrm{~cm}^{-1}\right)$ were recorded in $\mathrm{KBr}$ disk with the help of Perkin Elmer L-0100 and Nicolet Magna IR 750 Series II spectrometers. Proton NMR spectra were recorded on a Bruker FT $300 \mathrm{MHz}$ spectrometer. In ${ }^{1} \mathrm{H}$ NMR the spin-spin structures are abbreviated as follows: s, singlet; $d$, doublet; t, triplet: $\mathrm{m}$, multiplet and $\mathrm{b}$, broad. Electrochemical measurements were performed under nitrogen atmosphere on a $\mathrm{CH} 620 \mathrm{~A}$ electrochemical analyzer, using a platinum working electrode. 
The supporting electrolyte was tetraethylammonium perchlorate (TEAP) and the potentials are referred to the saturated calomel electrode (SCE) without junction correction. Microanalyses $(\mathrm{C}, \mathrm{H}, \mathrm{N})$ were performed using a Perkin-Elmer 2400 series II elemental analyzer. Solution electrical conductivity was measured in methanol $\left(\sim 10^{-3} \mathrm{M}\right.$ solution $)$ with a Phillips PR 9500 bridge using a platinized electrode (cell constant of 1.05). Roomtemperature magnetic susceptibilities of powders were measured with a model 155 PAR vibrating sample magnetometer.

\section{Synthesis}

\section{Synthesis of $\operatorname{Re}\left(\mathrm{OPPh} \mathrm{C}_{2}\left(\mathrm{CH}_{2}\right)_{x} \mathrm{PPh}_{2}\right) \mathrm{Cl}_{3}(\mathrm{dppz})$,(2)}

These were prepared by a general procedure: Reaction of $\mathrm{Re}^{\mathrm{V}} \mathrm{OCl}_{3}(\mathrm{dppz})$ with excess $\mathrm{Ph}_{2} \mathrm{P}\left(\mathrm{CH}_{2}\right)_{\mathrm{x}} \mathrm{PPh}_{2} \quad(\mathrm{x}=1-4)$ in dichloromethane solution. Details are given below for a representative case.

$\operatorname{Re}\left(\mathrm{OPPh}_{2}\left(\mathrm{CH}_{2}\right) \mathrm{PPh}_{2}\right) \mathrm{Cl}_{3}(\mathrm{dppz})$. To $65 \mathrm{mg} \quad(0.12 \mathrm{mmol})$ of complex $\mathbf{1}$ in dichloromethane solution was added $138 \mathrm{mg}(0.36 \mathrm{mmol})$ of $\mathrm{PPh}_{2}\left(\mathrm{CH}_{2}\right) \mathrm{PPh}_{2}$ and the solution was stirred magnetically at room temperature for $3 \mathrm{~h}$. During the reaction the solution color changed from orange yellow to violet. The solution was subjected to chromatography on a silica gel column $(25 \times 1 \mathrm{~cm}, 60-120 \mathrm{mesh})$. Excess diphosphine was eluted with benzene. The violet band that followed was eluted with a benzene-acetonitrile (25:1) mixture. Solvent removal from the eluate under reduced pressure afforded 2 as a violet solid. Yield: $78 \mathrm{mg}(70 \%)$. Anal. Calcd. for $\mathrm{C}_{39} \mathrm{H}_{32} \mathrm{Cl}_{3} \mathrm{~N}_{4} \mathrm{OP}_{2} \mathrm{Re}$ : C, 50.59; H, 3.48; $\mathrm{N}$, 5.94. Found: $\mathrm{C}, 50.61 ; \mathrm{H}, 3.51 ; \mathrm{N}, 5.89$. UV-vis $\left(\lambda_{\max }, \mathrm{nm}\left(\varepsilon, \mathrm{M}^{-1} \mathrm{~cm}^{-1}\right), \mathrm{CH}_{2} \mathrm{Cl}_{2}\right.$ solution): 738(4450), 578(2900), 475(5250). ${ }^{1} \mathrm{H}$ NMR $\delta$ in $\left(\mathrm{CDCl}_{3}\right)$ : dppz, 27.12(b, 1H), 23.56(d, 1H, J=7.2), 10.48(d, 1H, J=7.2 Hz), 9.92(t, 1H, J=8.4 Hz), 6.99(d, 1H, J=7.5 Hz),

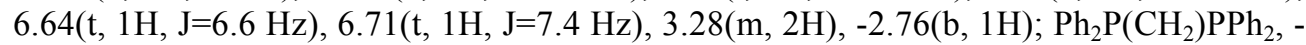
2.42(m, $\left.2 \mathrm{H}, \mathrm{CH}_{2}\right), 7.90(\mathrm{~m}, 4 \mathrm{H}), 7.86(\mathrm{~m}, 2 \mathrm{H}), 7.64(\mathrm{~m}, 4 \mathrm{H}), 7.38(\mathrm{~m}, 4 \mathrm{H}), 6.62(\mathrm{~m}, 2 \mathrm{H})$, 4.81(t, 2H, J=7.6 Hz), 4.73(t, 2H, J=8.1 Hz). IR ( $\mathrm{cm}^{-1}, \mathrm{KBr}$ disk): 304, 308, 326(Re-Cl), $1129(\mathrm{O}-\mathrm{P}), 1595(\mathrm{C}=\mathrm{N}) . \mathrm{E}_{1 / 2}$ (versus $\mathrm{SCE}, \mathrm{CH}_{3} \mathrm{CN}$, scan rate $\left.50 \mathrm{mV} \mathrm{s}{ }^{-1}\right): \mathrm{Re}^{\mathrm{IV}}-\mathrm{Re}^{\mathrm{III}}, 0.30$ $\mathrm{V}\left(\Delta \mathrm{E}_{\mathrm{p}}=80 \mathrm{mV}\right)$.

$\mathrm{Re}\left(\mathrm{OPPh}_{2}\left(\mathrm{CH}_{2}\right)_{2} \mathrm{PPh}_{2}\right) \mathrm{Cl}_{3}(\mathrm{dppz})$. Yield: $(70 \%)$. Anal. Calcd. for $\mathrm{C}_{40} \mathrm{H}_{34} \mathrm{Cl}_{3} \mathrm{~N}_{4} \mathrm{OP}_{2} \mathrm{Re}: \mathrm{C}$, 50.92; H, 3.63; N, 5.94. Found: C, 50.93; H, 3.65; N, 5.94. UV-vis $\left(\lambda_{\max }, \mathrm{nm}\left(\varepsilon, \mathrm{M}^{-1} \mathrm{~cm}^{-1}\right)\right.$, $\mathrm{CH}_{2} \mathrm{Cl}_{2}$ solution): 730(5500), 580(3100), 475(5800). ${ }^{1} \mathrm{H}$ NMR $\delta$ in $\left(\mathrm{CDCl}_{3}\right)$ : dppz, 25.34(b, $1 \mathrm{H}), 23.96(\mathrm{~d}, 1 \mathrm{H}, \mathrm{J}=7.7), 10.71(\mathrm{~d}, 1 \mathrm{H}, \mathrm{J}=7.8 \mathrm{~Hz}), 9.98(\mathrm{t}, 1 \mathrm{H}, \mathrm{J}=8.5 \mathrm{~Hz}), 6.78(\mathrm{t}, 1 \mathrm{H}$, $\mathrm{J}=7.8 \mathrm{~Hz}), 6.68(\mathrm{~d}, 1 \mathrm{H}, \mathrm{J}=6.8 \mathrm{~Hz}), 6.65(\mathrm{t}, 1 \mathrm{H}, \mathrm{J}=7.9 \mathrm{~Hz}), 2.45(\mathrm{~m}, 2 \mathrm{H}),-3.54(\mathrm{~b}, 1 \mathrm{H})$; $\mathrm{Ph}_{2} \mathrm{P}\left(\mathrm{CH}_{2}\right)_{2} \mathrm{PPh}_{2},-9.40\left(\mathrm{~s}, 2 \mathrm{H}, \mathrm{CH}_{2}\right),-2.36\left(\mathrm{~m}, 2 \mathrm{H}, \mathrm{CH}_{2}\right), 7.78(\mathrm{~m}, 4 \mathrm{H}), 7.48(\mathrm{~m}, 4 \mathrm{H}), 7.28(\mathrm{~m}$, $2 \mathrm{H}), 7.10(\mathrm{~m}, 4 \mathrm{H}), 6.45(\mathrm{~m}, 2 \mathrm{H}), 4.64(\mathrm{t}, 2 \mathrm{H}, \mathrm{J}=7.8 \mathrm{~Hz}), 4.32(\mathrm{t}, 2 \mathrm{H}, \mathrm{J}=8.1 \mathrm{~Hz}) . \mathrm{IR}\left(\mathrm{cm}^{-1}, \mathrm{KBr}\right.$ disk): $302,310,330(\mathrm{Re}-\mathrm{Cl}), 1127(\mathrm{O}-\mathrm{P}), 1594(\mathrm{C}=\mathrm{N}) . \mathrm{E}_{1 / 2}$ (versus $\mathrm{SCE}, \mathrm{CH}_{3} \mathrm{CN}$, scan rate 50 $\left.\mathrm{mV} \mathrm{s}^{-1}\right): \mathrm{Re}^{\mathrm{IV}}-\mathrm{Re}^{\mathrm{III}}, 0.29 \mathrm{~V}\left(\Delta \mathrm{E}_{\mathrm{p}}=80 \mathrm{mV}\right)$.

$\mathrm{Re}\left(\mathrm{OPPh}_{2}\left(\mathrm{CH}_{2}\right)_{3} \mathrm{PPh}_{2}\right) \mathrm{Cl}_{3}(\mathrm{dppz})$. Yield: $70 \%$. Anal. Calcd. for $\mathrm{C}_{41} \mathrm{H}_{36} \mathrm{Cl}_{3} \mathrm{~N}_{4} \mathrm{OP}_{2} \mathrm{Re}: \mathrm{C}$, 51.70; H, 3.81; N, 5.88. Found: C, 51.73; H, 3.80; N, 5.92. UV-vis $\left(\lambda_{\max }, \mathrm{nm}\left(\varepsilon, \mathrm{M}^{-1} \mathrm{~cm}^{-1}\right)\right.$, $\mathrm{CH}_{2} \mathrm{Cl}_{2}$ solution): 736(4950), 578(2950), 470(5500). ${ }^{1} \mathrm{H}$ NMR $\delta$ in $\left(\mathrm{CDCl}_{3}\right)$ : dppz, 24.90(b, $1 \mathrm{H}), 23.68(\mathrm{~d}, 1 \mathrm{H}, \mathrm{J}=7.8), 10.99(\mathrm{~d}, 1 \mathrm{H}, \mathrm{J}=7.6 \mathrm{~Hz}), 9.70(\mathrm{t}, 1 \mathrm{H}, \mathrm{J}=8.4 \mathrm{~Hz}), 6.98(\mathrm{~d}, 1 \mathrm{H}, \mathrm{J}=7.2$ $\mathrm{Hz}), \quad 6.78(\mathrm{t}, 1 \mathrm{H}, \mathrm{J}=7.6 \mathrm{~Hz}), \quad 6.50(\mathrm{t}, 1 \mathrm{H}, \mathrm{J}=7.6 \mathrm{~Hz}), \quad 3.40(\mathrm{~m}, 2 \mathrm{H}),-3.58(\mathrm{~b}, 1 \mathrm{H})$; $\mathrm{Ph}_{2} \mathrm{P}\left(\mathrm{CH}_{2}\right)_{3} \mathrm{PPh}_{2},-9.54\left(\mathrm{~s}, 2 \mathrm{H}, \mathrm{CH}_{2}\right),-4.48\left(\mathrm{~m}, 2 \mathrm{H}, \mathrm{CH}_{2}\right),-2.36\left(\mathrm{~m}, 2 \mathrm{H}, \mathrm{CH}_{2}\right), \quad 7.98(\mathrm{~m}, 4 \mathrm{H})$, 7.88(m, 4H), 7.42(m, 2H), 7.26(m, 4H), 6.32(m, 2H), 4.82(t, $2 \mathrm{H}, \mathrm{J}=7.8 \mathrm{~Hz}), 4.36(\mathrm{t}, 2 \mathrm{H}$, $\mathrm{J}=8.0 \mathrm{~Hz})$. IR ( $\mathrm{cm}^{-1}, \mathrm{KBr}$ disk): 303, 310, 328(Re-Cl), $1129(\mathrm{O}-\mathrm{P}), 1594(\mathrm{C}=\mathrm{N}) . \mathrm{E}_{1 / 2}$ (versus $\mathrm{SCE}, \mathrm{CH}_{3} \mathrm{CN}$, scan rate $\left.50 \mathrm{mV} \mathrm{s}^{-1}\right): \mathrm{Re}^{\mathrm{IV}}-\mathrm{Re}^{\mathrm{III}}, 0.30 \mathrm{~V}\left(\Delta \mathrm{E}_{\mathrm{p}}=80 \mathrm{mV}\right)$. 
$\mathrm{Re}\left(\mathrm{OPPh}_{2}\left(\mathrm{CH}_{2}\right)_{4} \mathrm{PPh}_{2}\right) \mathrm{Cl}_{3}$ (dppz). Yield: $70 \%$. Anal. Calcd. for $\mathrm{C}_{42} \mathrm{H}_{38} \mathrm{Cl}_{3} \mathrm{~N}_{4} \mathrm{OP}_{2} \mathrm{Re}: \mathrm{C}$, 51.96; H, 3.95; N, 5.77. Found: C, 51.93; H, 3.99; N, 5.82. UV-vis $\left(\lambda_{\max }, \mathrm{nm}\left(\varepsilon, \mathrm{M}^{-1} \mathrm{~cm}^{-1}\right)\right.$, $\mathrm{CH}_{2} \mathrm{Cl}_{2}$ solution): 732(4500), 586(2800), 480(5000). ${ }^{1} \mathrm{H}$ NMR $\delta$ in $\left(\mathrm{CDCl}_{3}\right): \mathrm{dppz}, 26.80(\mathrm{~b}$, $1 \mathrm{H}), 24.78(\mathrm{~d}, 1 \mathrm{H}, \mathrm{J}=7.8), 10.92(\mathrm{~d}, 1 \mathrm{H}, \mathrm{J}=8.4 \mathrm{~Hz}), 9.78(\mathrm{t}, 1 \mathrm{H}, \mathrm{J}=8.6 \mathrm{~Hz}), 6.96(\mathrm{~d}, 1 \mathrm{H}, \mathrm{J}=7.4$ $\mathrm{Hz}), \quad 6.59(\mathrm{t}, \quad 1 \mathrm{H}, \quad \mathrm{J}=7.4 \mathrm{~Hz}), \quad 6.49(\mathrm{t}, \quad 1 \mathrm{H}, \mathrm{J}=7.8 \mathrm{~Hz}), \quad 3.55(\mathrm{~m}, 2 \mathrm{H}),-4.63(\mathrm{~b}, 1 \mathrm{H})$; $\mathrm{Ph}_{2} \mathrm{P}\left(\mathrm{CH}_{2}\right)_{4} \mathrm{PPh}_{2},-10.59\left(\mathrm{~s}, 2 \mathrm{H}, \mathrm{CH}_{2}\right),-5.60\left(\mathrm{~m}, 2 \mathrm{H}, \mathrm{CH}_{2}\right),-2.81\left(\mathrm{~m}, 2 \mathrm{H}, \mathrm{CH}_{2}\right), 7.78(\mathrm{~m}, 4 \mathrm{H})$, $7.58(\mathrm{~m}, 4 \mathrm{H}), 7.37(\mathrm{~m}, 2 \mathrm{H}), 7.14(\mathrm{~m}, 4 \mathrm{H}), 6.34(\mathrm{~m}, 2 \mathrm{H}), 4.76(\mathrm{t}, 2 \mathrm{H}, \mathrm{J}=7.8 \mathrm{~Hz}), 3.24(\mathrm{t}, 2 \mathrm{H}$, $\mathrm{J}=8.2 \mathrm{~Hz}) . \mathrm{IR}\left(\mathrm{cm}^{-1}, \mathrm{KBr}\right.$ disk): $305,311,332(\mathrm{Re}-\mathrm{Cl}), 1130(\mathrm{O}-\mathrm{P}), 1594(\mathrm{C}=\mathrm{N}) . \mathrm{E}_{1 / 2}$ (versus $\mathrm{SCE}, \mathrm{CH}_{3} \mathrm{CN}$, scan rate $\left.50 \mathrm{mV} \mathrm{s}^{-1}\right): \mathrm{Re}^{\mathrm{IV}}-\mathrm{Re}^{\mathrm{III}}, 0.30 \mathrm{~V}\left(\Delta \mathrm{E}_{\mathrm{p}}=80 \mathrm{mV}\right)$.

\section{Synthesis of $\operatorname{Re}\left(\mathrm{PPh}_{2}\left(\mathrm{CH}_{2}\right)_{x} \mathrm{P}(\mathrm{O}) \mathrm{Ph}_{2}\right) \mathrm{Cl}_{3}(\mathrm{dppz})$,(3)}

The general procedure consisted of simply leaving a dichloromethane solution of $\operatorname{Re}\left(\mathrm{OPPh}_{2}\left(\mathrm{CH}_{2}\right)_{\mathrm{x}} \mathrm{PPh}_{2}\right) \mathrm{Cl}_{3}(\mathrm{dppz})$ to isomerizes at room temperature $(\approx 298 \mathrm{~K})$ in a stoppered flask for 1,2, 4 and 5 days for the cases of $\mathrm{x}=1,2,3,4$ respectively. Procedural details are given below for a representative case.

$\mathrm{Re}\left(\mathrm{PPh}_{2}\left(\mathrm{CH}_{2}\right) \mathrm{P}(\mathrm{O}) \mathrm{Ph}_{2}\right) \mathrm{Cl}_{3}(\mathrm{dppz})$, A $69 \mathrm{mg}(0.08 \mathrm{mmol})$ of $\mathrm{Re}\left(\mathrm{OPPh}_{2}\left(\mathrm{CH}_{2}\right) \mathrm{PPh}_{2}\right) \mathrm{Cl}_{3}$ (dppz) was dissolved in $25 \mathrm{~mL}$ dichloromethane and the solution was left for $24 \mathrm{~h}$. It was then subjected to chromatography on a silica gel column. A green band was eluted with a benzene-acetonitrile $(25: 10)$ mixture. Solvent removal under reduced pressure afforded $\mathrm{Re}\left(\mathrm{PPh}_{2}\left(\mathrm{CH}_{2}\right) \mathrm{P}(\mathrm{O}) \mathrm{Ph}_{2}\right) \mathrm{Cl}_{3}(\mathrm{dppz})$ as a green solid which was dried under vacuum over fused $\mathrm{CaCl}_{2}$. Yield: $62 \mathrm{mg}(85 \%)$. Anal. Calcd. For $\mathrm{C}_{39} \mathrm{H}_{32} \mathrm{Cl}_{3} \mathrm{~N}_{4} \mathrm{OP}_{2} \mathrm{Re}$ : C, 50.59; H, 3.48; N, 6.05. Found: $\mathrm{C}, 50.55 ; \mathrm{H}, 3.49 ; \mathrm{N}, 6.08$. UV-vis $\left(\lambda_{\max }, \mathrm{nm}\left(\varepsilon, \mathrm{M}^{-1} \mathrm{~cm}^{-1}\right), \mathrm{CH}_{2} \mathrm{Cl}_{2}\right.$ solution): 660(1600), 590 $(1500), 467^{\mathrm{s}}(2750), 315(22800) .{ }^{1} \mathrm{H}$ NMR $\delta$ in $\left(\mathrm{CDCl}_{3}\right): \mathrm{dppz}, 20.12(\mathrm{t}, 1 \mathrm{H}$, $\mathrm{J}=8.4 \mathrm{~Hz}), 19.46(\mathrm{~d}, 1 \mathrm{H}, \mathrm{J}=7.8 \mathrm{~Hz}), 15.76(\mathrm{~d}, 1 \mathrm{H}, \mathrm{J}=6.9 \mathrm{~Hz}), 7.86(\mathrm{t}, 1 \mathrm{H}, \mathrm{J}=7.8 \mathrm{~Hz}), 6.82(\mathrm{~d}$, $1 \mathrm{H}, \mathrm{J}=8.6 \mathrm{~Hz}), 5.82(\mathrm{t}, 1 \mathrm{H}, \mathrm{J}=7.6 \mathrm{~Hz}), 3.56(\mathrm{~m}, 2 \mathrm{H}), 0.40(\mathrm{t}, 1 \mathrm{H}, \mathrm{J}=7.2 \mathrm{~Hz}),-3.86(\mathrm{~d}, 1 \mathrm{H}, \mathrm{J}=6.2$ $\mathrm{Hz}) ; \mathrm{Ph}_{2} \mathrm{P}\left(\mathrm{CH}_{2}\right) \mathrm{PPh}_{2},-2.86\left(\mathrm{~m}, 2 \mathrm{H}, \mathrm{CH}_{2}\right), 17.94(\mathrm{~m}, 2 \mathrm{H}), 16.98(\mathrm{~m}, 2 \mathrm{H}), 8.41(\mathrm{~m}, 2 \mathrm{H}), 7.35(\mathrm{~m}$, $4 \mathrm{H}), 7.18(\mathrm{~m}, 4 \mathrm{H}), 6.56(\mathrm{~m}, 2 \mathrm{H}), 4.89(\mathrm{t}, 2 \mathrm{H}, \mathrm{J}=7.4 \mathrm{~Hz}), 4.56(\mathrm{t}, 2 \mathrm{H}, \mathrm{J}=8.4 \mathrm{~Hz}) . \mathrm{IR}\left(\mathrm{cm}^{-1}, \mathrm{KBr}\right.$ disk): 308, 319, 329(Re-Cl), 1193(P-O), $1595(\mathrm{C}=\mathrm{N}) . \mathrm{E}_{1 / 2}$ (versus $\mathrm{SCE}, \mathrm{CH}_{3} \mathrm{CN}$, scan rate 50 $\left.\mathrm{mV} \mathrm{s}^{-1}\right): \mathrm{Re}^{\mathrm{IV}}-\mathrm{Re}^{\mathrm{III}}, 0.60 \mathrm{~V}\left(\Delta \mathrm{E}_{\mathrm{p}}=80 \mathrm{mV}\right)$.

$\mathrm{Re}\left(\mathrm{PPh}_{2}\left(\mathrm{CH}_{2}\right)_{2} \mathrm{P}(\mathrm{O}) \mathrm{Ph}_{2}\right) \mathrm{Cl}_{3}(\mathrm{dppz})$, Yield: $83 \%$. Anal. Calcd. for $\mathrm{C}_{40} \mathrm{H}_{34} \mathrm{Cl}_{3} \mathrm{~N}_{4} \mathrm{OP}_{2} \mathrm{Re}: \mathrm{C}$, 50.92; H, 3.63; N, 5.94. Found: C, 50.95; H, 3.59; N, 5.93. UV-vis $\left(\lambda_{\max }, \mathrm{nm}\left(\varepsilon, \mathrm{M}^{-1} \mathrm{~cm}^{-1}\right)\right.$, $\mathrm{CH}_{2} \mathrm{Cl}_{2}$ solution): 669(1550), 593 ${ }^{\mathrm{s}}(1650), 461^{\mathrm{s}}(2600), 315(22000) .{ }^{1} \mathrm{H}$ NMR $\delta$ in $\left(\mathrm{CDCl}_{3}\right)$ : dppz, 22.58(d, 1H, J=8.2 Hz), 19.31(t, 1H, J=7.5 Hz), 15.90(d, 1H, J=6.6 Hz), 7.78(t, 1H, $\mathrm{J}=7.4 \mathrm{~Hz}), 6.80(\mathrm{~d}, 1 \mathrm{H}, \mathrm{J}=8.0 \mathrm{~Hz}), 5.99(\mathrm{t}, 1 \mathrm{H}, \mathrm{J}=7.6 \mathrm{~Hz}), 3.34(\mathrm{~m}, 2 \mathrm{H}), 1.40(\mathrm{t}, 1 \mathrm{H}, \mathrm{J}=6.8 \mathrm{~Hz}),-$ 2.86(d, $1 \mathrm{H}, \mathrm{J}=6.4 \mathrm{~Hz}) ; \mathrm{Ph}_{2} \mathrm{P}\left(\mathrm{CH}_{2}\right)_{2} \mathrm{PPh}_{2},-5.58\left(\mathrm{~m}, 2 \mathrm{H}, \mathrm{CH}_{2}\right),-3.36\left(\mathrm{~m}, 2 \mathrm{H}, \mathrm{CH}_{2}\right), 18.48(\mathrm{~m}, 2 \mathrm{H})$, 17.80(m, 2H), 8.20(m, 2H), 7.66(m, 4H), 7.36(m, 4H), 6.36(m, 2H), 4.59(t, 2H, J=7.2 Hz), 4.26(t, 2H, J=8.6 Hz). IR ( $\mathrm{cm}^{-1}, \mathrm{KBr}$ disk $): 310,320,330(\mathrm{Re}-\mathrm{Cl}), 1186(\mathrm{P}-\mathrm{O}), 1590(\mathrm{C}=\mathrm{N}) . \mathrm{E}_{1 / 2}$ (versus SCE, $\mathrm{CH}_{3} \mathrm{CN}$, scan rate $\left.50 \mathrm{mV} \mathrm{s}^{-1}\right): \mathrm{Re}^{\mathrm{IV}}-\mathrm{Re}^{\mathrm{III}}, 0.61 \mathrm{~V}\left(\Delta \mathrm{E}_{\mathrm{p}}=80 \mathrm{mV}\right)$.

$\mathrm{Re}\left(\mathrm{PPh}_{2}\left(\mathrm{CH}_{2}\right)_{3} \mathrm{P}(\mathrm{O}) \mathrm{Ph}_{2}\right) \mathrm{Cl}_{3}(\mathrm{dppz})$, Yield: $80 \%$. Anal. Calcd. for $\mathrm{C}_{41} \mathrm{H}_{36} \mathrm{Cl}_{3} \mathrm{~N}_{4} \mathrm{OP}_{2} \mathrm{Re}: \mathrm{C}$, 51.70; H, 3.81; N, 5.88. Found: C, 50.71; H, 3.85; N, 5.87. UV-vis $\left(\lambda_{\max }, \mathrm{nm}\left(\varepsilon, \mathrm{M}^{-1} \mathrm{~cm}^{-1}\right)\right.$, $\mathrm{CH}_{2} \mathrm{Cl}_{2}$ solution): 666(1700), 590 ${ }^{\mathrm{s}}(1800), 465^{\mathrm{s}}(2950), 315(27000) .{ }^{1} \mathrm{H}$ NMR $\delta$ in $\left(\mathrm{CDCl}_{3}\right)$ : dppz, 22.36(d, 1H, J=8.4 Hz), 19.90(t, 1H, J=7.6 Hz), 15.80(d, 1H, J=6.8 Hz), 7.82(t, 1H, J=7.5 $\mathrm{Hz}), 6.99(\mathrm{~d}, 1 \mathrm{H}, \mathrm{J}=8.6 \mathrm{~Hz}), 5.89(\mathrm{t}, 1 \mathrm{H}, \mathrm{J}=7.4 \mathrm{~Hz}), 3.44(\mathrm{~m}, 2 \mathrm{H}), 1.98(\mathrm{t}, 1 \mathrm{H}, \mathrm{J}=6.4 \mathrm{~Hz}),-2.75(\mathrm{~d}$, $1 \mathrm{H}, \mathrm{J}=6.4 \mathrm{~Hz}) ; \mathrm{Ph}_{2} \mathrm{P}\left(\mathrm{CH}_{2}\right)_{3} \mathrm{PPh}_{2},-8.66\left(\mathrm{~m}, 2 \mathrm{H}, \mathrm{CH}_{2}\right),-5.42\left(\mathrm{~m}, 2 \mathrm{H}, \mathrm{CH}_{2}\right),-3.56\left(\mathrm{~m}, 2 \mathrm{H}, \mathrm{CH}_{2}\right)$, 17.26(m, 2H), 16.98(m, 2H), 8.38(m, 2H), 7.42(m, 4H), 7.28(m, 4H), 6.45(m, 2H), 4.48(t, 2H, $\mathrm{J}=7.8 \mathrm{~Hz}), 4.16(\mathrm{t}, 2 \mathrm{H}, \mathrm{J}=8.2 \mathrm{~Hz})$. IR ( $\mathrm{cm}^{-1}, \mathrm{KBr}$ disk): 310, 324, 331(Re-Cl), 1190(P-O), $1592(\mathrm{C}=\mathrm{N}) . \mathrm{E}_{1 / 2}$ (versus $\mathrm{SCE}, \mathrm{CH}_{3} \mathrm{CN}$, scan rate $\left.50 \mathrm{mV} \mathrm{s}^{-1}\right)$ : $\mathrm{Re}^{\mathrm{IV}}-\mathrm{Re}^{\mathrm{III}}, 0.59 \mathrm{~V}\left(\Delta \mathrm{E}_{\mathrm{p}}=80 \mathrm{mV}\right.$ ). 
$\mathrm{Re}\left(\mathrm{PPh}_{2}\left(\mathrm{CH}_{2}\right)_{4} \mathrm{P}(\mathrm{O}) \mathrm{Ph}_{2}\right) \mathrm{Cl}_{3}(\mathrm{dppz})$, Yield: $80 \%$. Anal. Calcd. for $\mathrm{C}_{42} \mathrm{H}_{38} \mathrm{Cl}_{3} \mathrm{~N}_{4} \mathrm{OP}_{2} \mathrm{Re}: \mathrm{C}$, 51.96; H, 3.95; N, 5.77. Found: C, 51.96; H, 3.95; N, 5.77. UV-vis $\left(\lambda_{\max }, \mathrm{nm}\left(\varepsilon, \mathrm{M}^{-1} \mathrm{~cm}^{-1}\right), \mathrm{CH}_{2} \mathrm{Cl}_{2}\right.$ solution): 667(1800), 589 $(1900), 462^{\mathrm{s}}(3100), 320(26000) .{ }^{1} \mathrm{H}$ NMR $\delta$ in $\left(\mathrm{CDCl}_{3}\right)$ : dppz, $22.67(\mathrm{~d}$, $1 \mathrm{H}, \mathrm{J}=8.6 \mathrm{~Hz}), 21.88(\mathrm{t}, 1 \mathrm{H}, \mathrm{J}=7.8 \mathrm{~Hz}), 15.89(\mathrm{~d}, 1 \mathrm{H}, \mathrm{J}=6.9 \mathrm{~Hz}), 7.95(\mathrm{t}, 1 \mathrm{H}, \mathrm{J}=8.2 \mathrm{~Hz}), 6.96(\mathrm{~d}, 1 \mathrm{H}$, $\mathrm{J}=8.4 \mathrm{~Hz}), 5.85(\mathrm{t}, 1 \mathrm{H}, \mathrm{J}=7.8 \mathrm{~Hz}), 3.66(\mathrm{~m}, 2 \mathrm{H}), 1.68(\mathrm{t}, 1 \mathrm{H}, \mathrm{J}=6.8 \mathrm{~Hz}),-2.48(\mathrm{~d}, 1 \mathrm{H}, \mathrm{J}=6.9 \mathrm{~Hz})$; $\mathrm{Ph}_{2} \mathrm{P}\left(\mathrm{CH}_{2}\right)_{4} \mathrm{PPh}_{2},-9.64\left(\mathrm{~m}, 2 \mathrm{H}, \mathrm{CH}_{2}\right),-8.48\left(\mathrm{~m}, 2 \mathrm{H}, \mathrm{CH}_{2}\right),-5.69\left(\mathrm{~m}, 2 \mathrm{H}, \mathrm{CH}_{2}\right),-3.49\left(\mathrm{~m}, 2 \mathrm{H}, \mathrm{CH}_{2}\right)$, 17.34(m, 2H), 16.80(m, 2H), 8.76(m, 2H), 7.42(m, 4H), 7.23(m, 4H), 6.38(m, 2H), 4.68(t, $2 \mathrm{H}$, $\mathrm{J}=8.4 \mathrm{~Hz}), 4.48(\mathrm{t}, 2 \mathrm{H}, \mathrm{J}=8.4 \mathrm{~Hz}) . \mathrm{IR}\left(\mathrm{cm}^{-1}, \mathrm{KBr}\right.$ disk): 311, 322, 330(Re-Cl), 1191(P-O), $1594(\mathrm{C}=\mathrm{N}) . \mathrm{E}_{1 / 2}$ (versus $\mathrm{SCE}, \mathrm{CH}_{3} \mathrm{CN}$, scan rate $\left.50 \mathrm{mV} \mathrm{s}^{-1}\right): \mathrm{Re}^{\mathrm{IV}}-\mathrm{Re}^{\mathrm{III}}, 0.60 \mathrm{~V}\left(\Delta \mathrm{E}_{\mathrm{p}}=80 \mathrm{mV}\right)$.

\section{Synthesis of $\operatorname{Re}\left(\mathrm{OPPh}_{2}\left(\mathrm{CH}_{2}\right) \mathrm{P}(\mathrm{O}) \mathrm{Ph}_{2}\right) \mathrm{Cl}_{3}(\mathrm{dppz}),(4)$}

To $52 \mathrm{mg}(0.06 \mathrm{mmol})$ of complex $\operatorname{Re}\left(\mathrm{OPPh}_{2}\left(\mathrm{CH}_{2}\right) \mathrm{PPh}_{2}\right) \mathrm{Cl}_{3}(\mathrm{dppz})$ in dichloromethane solution $33 \mathrm{mg}(0.06 \mathrm{mmol})$ of $\mathbf{1}$ was added and the solution was stirred magnetically at room temperature for $3 \mathrm{~h}$. The solution was then subjected to chromatography on a silica gel column $(25 \times 1 \mathrm{~cm}, 60-120$ mesh). A violet band was eluted with a benzeneacetonitrile (25:1) mixture. Solvent removal from the eluate under reduced pressure afforded a violet solid. The product was dissolved in minimum amount of dichloromethane and was subjected to thin layer chromatography with a benzeneacetonitrile $(25: 1)$ mixture yielding two violet bands. The lower band was separated and extracted with benzene-acetonitrile (25:1) mixture. Solvent removal from the eluate under reduced pressure afforded 4 as a violet solid. Yield: $27 \mathrm{mg}$ (51\%). Anal. Calcd. for $\mathrm{C}_{39} \mathrm{H}_{32} \mathrm{Cl}_{3} \mathrm{~N}_{4} \mathrm{O}_{2} \mathrm{P}_{2}$ Re: $\mathrm{C}, 49.65 ; \mathrm{H}, 3.42 ; \mathrm{N}, 5.94$. Found: $\mathrm{C}, 49.65 ; \mathrm{H}, 3.47 ; \mathrm{N}, 5.95$. UVvis $\left(\lambda_{\max }, \mathrm{nm}\left(\varepsilon, \mathrm{M}^{-1} \mathrm{~cm}^{-1}\right), \mathrm{CH}_{2} \mathrm{Cl}_{2}\right.$ solution): 730(5500), 595(2950), 480(5050). ${ }^{1} \mathrm{H} \mathrm{NMR}$ $\delta$ in $\left(\mathrm{CDCl}_{3}\right)$ : dppz, 22.56(b, 1H), 21.51(d, $\left.1 \mathrm{H}, \mathrm{J}=7.6\right), 10.65(\mathrm{~d}, 1 \mathrm{H}, \mathrm{J}=7.8 \mathrm{~Hz}), 9.78(\mathrm{t}, 1 \mathrm{H}$, $\mathrm{J}=8.2 \mathrm{~Hz}), 6.96(\mathrm{~d}, 1 \mathrm{H}, \mathrm{J}=7.6 \mathrm{~Hz}), 6.88(\mathrm{t}, 1 \mathrm{H}, \mathrm{J}=6.9 \mathrm{~Hz}), 6.68(\mathrm{t}, 1 \mathrm{H}, \mathrm{J}=7.8 \mathrm{~Hz}), 3.66(\mathrm{~m}$, 2H), -5.78(b, 1H); $\mathrm{Ph}_{2} \mathrm{P}\left(\mathrm{CH}_{2}\right) \mathrm{PPh}_{2},-4.38\left(\mathrm{~m}, 2 \mathrm{H}, \mathrm{CH}_{2}\right), 7.94(\mathrm{~m}, 4 \mathrm{H}), 7.84(\mathrm{~m}, 2 \mathrm{H}), 7.51(\mathrm{~m}$, $4 \mathrm{H}), 7.25(\mathrm{~m}, 4 \mathrm{H}), 6.22(\mathrm{~m}, 2 \mathrm{H}), 4.75(\mathrm{t}, 2 \mathrm{H}, \mathrm{J}=7.8 \mathrm{~Hz}), 4.56(\mathrm{t}, 2 \mathrm{H}, \mathrm{J}=8.4 \mathrm{~Hz}) . \mathrm{IR}\left(\mathrm{cm}^{-1}\right.$, $\mathrm{KBr}$ disk): 305, 309, 328(Re-Cl), 1129(O-P), 1190(P-O), 1596(C=N). $\mathrm{E}_{1 / 2}$ (versus SCE, $\mathrm{CH}_{3} \mathrm{CN}$, scan rate $\left.50 \mathrm{mV} \mathrm{s}^{-1}\right)$ : $\mathrm{Re}^{\mathrm{IV}}-\mathrm{Re}^{\mathrm{III}}, 0.31 \mathrm{~V}\left(\Delta \mathrm{E}_{\mathrm{p}}=80 \mathrm{mV}\right)$.

\section{Synthesis of (dppz) $\mathrm{Cl}_{3} \operatorname{Re}\left(\mathrm{OPPh} \mathrm{C}_{2}\left(\mathrm{CH}_{2}\right)_{x} \mathrm{Ph}_{2} \mathrm{PO}\right) \mathrm{ReCl}_{3}(\mathrm{dppz}),(5)(x=2-4)$}

These were prepared by a general procedure: reaction of $\mathrm{Re}^{\mathrm{V}} \mathrm{OCl}_{3}(\mathrm{dppz})$ with $\mathrm{Re}\left(\mathrm{OPPh}_{2}\left(\mathrm{CH}_{2}\right)_{\mathrm{x}} \mathrm{PPh}_{2}\right) \mathrm{Cl}_{3}(\mathrm{dppz})(\mathrm{x}=2-4)$ in dichloromethane solution. Details are given below for a representative case.

\section{Synthesis of (dppz) $\left.\mathrm{Cl}_{3} \mathrm{Re}\left(\mathrm{OPPh} \mathrm{CH}_{2}\right)_{2} \mathrm{Ph}_{2} \mathrm{PO}\right) \mathrm{ReCl}_{3}(\mathrm{dppz})$}

To $33 \mathrm{mg}(0.06 \mathrm{mmol})$ of complex 1 in dichloromethane solution $53 \mathrm{mg}(0.06 \mathrm{mmol})$ of $\mathrm{Re}\left(\mathrm{OPPh}_{2}\left(\mathrm{CH}_{2}\right)_{2} \mathrm{PPh}_{2}\right) \mathrm{Cl}_{3}(\mathrm{dppz})$ was added and the solution was stirred magnetically at room temperature for $3 \mathrm{~h}$. During the reaction a violet precipitate separated from the solution was collected by filtration, washed thoroughly with dichloromethane and dried in vacuum over fused $\mathrm{CaCl}_{2}$. The precipitate was found to be insoluble in all common organic solvents and thus could only be characterized by elemental analysis and IR spectra. Yield: $52 \mathrm{mg}(60 \%)$. Anal. Calcd. for $\mathrm{C}_{54} \mathrm{H}_{44} \mathrm{Cl}_{6} \mathrm{~N}_{4} \mathrm{O}_{2} \mathrm{P}_{2} \mathrm{Re}_{2}$ : C, 45.40; H, 3.11; N, 3.92. Found: C, 45.37; H, 3.18; N, 3.86. IR ( $\mathrm{cm}^{-1}$, $\mathrm{KBr}$ disk): 305, 307, 309, 311, 326, 328( $\mathrm{Re}-\mathrm{Cl}), 1128,1131(\mathrm{O}-\mathrm{P}), 1596(\mathrm{C}=\mathrm{N})$.

\section{$\mathrm{Cl}_{3} \mathrm{Re}\left(\mathrm{OPPh} \mathrm{CH}_{2} \mathrm{CH}_{3} \mathrm{Ph}_{2} \mathrm{OP}\right) \mathrm{ReCl}_{3}(\mathrm{dppz})$}

Yield: $62 \%$. Anal. Calcd. for $\mathrm{C}_{55} \mathrm{H}_{46} \mathrm{Cl}_{6} \mathrm{~N}_{4} \mathrm{O}_{2} \mathrm{P}_{2} \mathrm{Re}_{2}: \mathrm{C}, 45.58 ; \mathrm{H}, 3.20 ; \mathrm{N}, 3.87$. Found: $\mathrm{C}$, 45.62; H, 3.18; N, 3.79. IR (cm ${ }^{-1}, \mathrm{KBr}$ disk): 304, 309, 312, 325, 329, 331(Re-Cl), 1126, 1132(O-P), 1592(C=N). 


\section{$\mathrm{Cl}_{3} \mathrm{Re}\left(\mathrm{OPPh} \mathrm{C}_{2}\left(\mathrm{CH}_{2}\right)_{4} \mathrm{Ph}_{2} \mathrm{OP}\right) \mathrm{ReCl}_{3}(\mathrm{dppz})$}

Yield: $65 \%$. Anal. Calcd. For $\mathrm{C}_{56} \mathrm{H}_{48} \mathrm{Cl}_{6} \mathrm{~N}_{4} \mathrm{O}_{2} \mathrm{P}_{2} \mathrm{Re}_{2}$ : C, 46.41; H, 3.34; N, 3.87. Found: C, 46.39; H, 3.29; N, 3.89. IR (cm ${ }^{-1}, \mathrm{KBr}$ disk): 302, 305, 310, 326, 328, 333(Re-Cl), 1128, $1131(\mathrm{O}-\mathrm{P}), 1591(\mathrm{C}=\mathrm{N})$.

Synthesis of $\left[\mathrm{Re}\left(\mathrm{OPPh}_{2}\left(\mathrm{CH}_{2}\right) \mathrm{PPh}_{2}\right) \mathrm{Cl}_{3}(\mathrm{dppz})\right] \mathrm{ReO}_{4}$, (6)

To $69 \mathrm{mg}(0.08 \mathrm{mmol})$ of $\mathrm{Re}\left(\mathrm{OPPh}_{2}\left(\mathrm{CH}_{2}\right) \mathrm{PPh}_{2}\right) \mathrm{Cl}_{3}(\mathrm{dppz})$ in $20 \mathrm{~mL}$ acetonitrile dilute aqueous $\mathrm{HNO}_{3}$ was added and the solution was stirred at room temperature for $0.25 \mathrm{~h}$. During this time the solution color changed from violet to yellow. Solvent removal under reduced pressure afforded $\left[\mathrm{Re}\left(\mathrm{OPPh}_{2}\left(\mathrm{CH}_{2}\right) \mathrm{PPh}_{2}\right) \mathrm{Cl}_{3}(\mathrm{dppz})\right] \mathrm{ReO}_{4}$ as an yellow solid. The solid was washed thoroughly with water to remove the adherent nitric acid and then dried in vacuum over fused $\mathrm{CaCl}_{2}$. Yield: $60 \mathrm{mg}(65 \%)$. Anal. Calcd. for $\mathrm{C}_{39} \mathrm{H}_{32} \mathrm{Cl}_{3} \mathrm{~N}_{4} \mathrm{O}_{5} \mathrm{P}_{2} \mathrm{Re}_{2}: \mathrm{C}$, 39.81; H, 2.74; N, 4.76. Found: C, 39.87; H, 2.75; N, 4.74. UV-vis $\left(\lambda \max , \mathrm{nm}\left(\varepsilon, \mathrm{M}^{-1} \mathrm{~cm}^{-1}\right)\right.$, $\mathrm{CH}_{2} \mathrm{Cl}_{2}$ solution): 425(800), 320(10600). ${ }^{1} \mathrm{H} \mathrm{NMR} \delta$ in $\left(\mathrm{CDCl}_{3}\right): \operatorname{dppz}, 74.28(1 \mathrm{H})$, $12.36(2 \mathrm{H}), \quad 8.78(1 \mathrm{H}), \quad 3.66(2 \mathrm{H}), \quad 2.99(2 \mathrm{H}), \quad-64.78(1 \mathrm{H}), \quad-91.19(1 \mathrm{H}) ; \quad \mathrm{PPh}_{2}\left(\mathrm{CH}_{2}\right) \mathrm{PPh}_{2}$, 9.18(2H), 7.86(4H), 7.68(4H), 7.54(4H), 6.54(4H), 5.84(2H), 4.86(2H). IR ( $\mathrm{cm}^{-1}, \mathrm{KBr}$ disk): 329, 334, 339(Re-Cl), 1117(O-P), 1596(C=N), 904(Re-O). $\mathrm{E}_{1 / 2}$ (versus $\mathrm{SCE}, \mathrm{CH}_{3} \mathrm{CN}$, scan rate $\left.50 \mathrm{mV} \mathrm{s}^{-1}\right): \operatorname{Re}^{\mathrm{IV}}-\mathrm{Re}^{\mathrm{III}}, 0.30 \mathrm{~V}\left(\Delta \mathrm{E}_{\mathrm{p}}=80 \mathrm{mV}\right) . \Lambda \mathrm{M}=97 \Omega^{-1} \mathrm{~cm}^{2} \mathrm{~mol}^{-1}$.

\section{Rate measurements}

The rate of the single oxygen atom transfer reaction of Eq. 2 was determined spectrophotometrically (quartz cell, path length $1 \mathrm{~cm}$ ) in the case of $\mathrm{Ph}_{2} \mathrm{P}\left(\mathrm{CH}_{2}\right)_{4} \mathrm{PPh}_{2}$. A known excess of the diphosphine was added to a solution of $\left[\mathrm{ReOCl}_{3}(\mathrm{dppz})\right]\left(1.25 \times 10^{-4}\right.$ $\mathrm{M})$ in dichloromethane at $308 \mathrm{~K}$ and the absorbance $A_{t}$ monitored for the peak at $732 \mathrm{~nm}$ as a function of time $(t)$. The time dependent spectra are characterised by isosbestic point. The absorbance $A_{\alpha}$ at $732 \mathrm{~nm}$ at the end of the reaction $(6 \mathrm{~h})$ was also monitored. The calculations were performed using Microcal Origin V 6.0 (E. Northampton, Microcal Origin Inc., 1991). The observed rate constants $k_{\text {obs }}$ were determined from the slope of the linear plot of $\ln \left(A_{\alpha}-A_{t}\right)$ versus $t$. The rate constant $k_{\text {oat }}$ was obtained from the linear plot of $k_{\text {obsd }}$ versus concentration of the diphosphine. The rate of the twin isomerization process of Eq. 3 was also followed spectrophotometrically at $308 \mathrm{~K}$. Time dependent absorbances $A_{t}$ were measured at $580 \mathrm{~nm}$ and $A_{\alpha}$ values were obtained at the end of the reaction (1 to 7 days depending on $x$ ). Rate constants were determined from the linear plots of $-\ln \left(A_{t^{-}} A_{\alpha}\right)$ versus $t$. Variable concentration $\left(1-4 \times 10^{-4} \mathrm{M}\right)$ studies carried out in the cases of $x=1$ revealed that the rate constants were independent of concentration consistent with first order kinetics. The plot of rate constants against diphosphine spacer length $(x)$ follows a single exponential decay pattern with reduced $\chi^{2}$ value of $\approx 10^{-11}$.

\section{Results and Discussion}

\section{Spectra}

In the visible region $(400-900 \mathrm{~nm})$ 2-4 display multiple transitions of moderate intensity $\left(\varepsilon, 1000-5000 \mathrm{dm}^{3} \mathrm{~mol}^{-1} \mathrm{~cm}^{-1}\right)$ in the form of peaks and shoulders. The most prominent feature occurs around $730 \mathrm{~nm}$ for 2 and around $660 \mathrm{~nm}$ for 3 . An idealized $t_{2 g}(\mathrm{Re}) \rightarrow \pi^{*}(\mathrm{dppz})$ MLCT assignment is consistent with the observed shift to higher energy in going from phosphine oxide 2 to the phosphine coordination 3 which stabilizes the $t_{2 g}$ shell via back-bonding. This is also reflected in the significant increase of the $\mathrm{Re}^{\mathrm{IV}} / \mathrm{Re}^{\mathrm{III}}$ reduction potentials. The rhenium(IV) species 6 in which MLCT transitions are expected to shift to much higher energies, does not display any band in the visible region except for a weak one at $425 \mathrm{~nm}$. 
Two or three Re-Cl stretches in the range $300-340 \mathrm{~cm}^{-1}$ occur in the complexes of type 2, 3, 4, 6 while more signals occur for dimeric type 5 complexes. The coordinated P-O stretch (2, $4,5)$ is observed near $1130 \mathrm{~cm}^{-1}$ as compared to $\approx 1190 \mathrm{~cm}^{-1}$ characterizing uncoordinated P-O $(3,4)^{7,10}$. In 6 the oxidized metal weakens the P-O bond and the vibration frequency drops to $1118 \mathrm{~cm}^{-1}$. A characteristic Re-O stretch of the $\mathrm{ReO}_{4}{ }^{-}$anion in 6 occurs near $904 \mathrm{~cm}^{-1}$.

The complexes of the type 2-4 have magnetic moment near $2 \mu \mathrm{B}$ as in several other rhenium(III) species ${ }^{4,6,7,8,11}$. All the complexes display paramagnetically shifted ${ }^{1} \mathrm{H}$ NMR lines. Well-resolved spin-spin structures are seen for 2-4 but not for $\mathbf{6}$.

\section{Metal redox}

The complexes uniformly display a nearly reversible $\mathrm{Re}^{\mathrm{IV}} / \mathrm{Re}^{\mathrm{III}}$ couple in acetonitrile solution, the cyclic voltammetric peak-to-peak separation being $60-80 \mathrm{mV}$. The reduction potentials lie near $0.30 \mathrm{~V}$ vs. SCE in 2 and 4 and near $0.60 \mathrm{~V}$ in 3 . The sizeable difference of reduction potentials makes it possible to observe the isomerization of $\mathbf{2}$ to $\mathbf{3}$ electrochemically as shown in Figure 1. Quantitative isomerization studies are however best made spectrophotometrically.

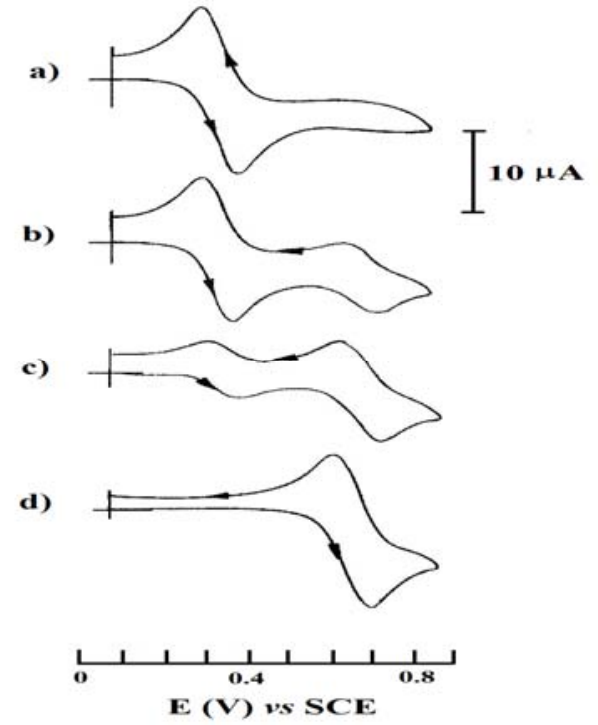

Figure 1. Cyclic voltammetric observation of the isomerization of $\operatorname{Re}\left(\mathrm{OPPh}_{2}\left(\mathrm{CH}_{2}\right) \mathrm{PPh}_{2}\right)$ $\mathrm{Cl}_{3}(\mathrm{dppz})$ in acetonitrile solution in the time domain 0-24 h : a) $\left.\left.0 \mathrm{~h}, \mathrm{~b}\right) 1.5 \mathrm{~h}, \mathrm{c}\right) 4 \mathrm{~h}$, d) $24 \mathrm{~h}$

The phosphine oxide ligand is a pure donor and optimal metal ligand bonding is achievable in the meridional geometry, there being no bonding advantage for the sterically unfavourable facial disposition of ligands. It is thus logical that 2, 4, 5 assumes meridional geometry. Upon substitution of $\mathrm{OPPh}_{2}\left(\mathrm{CH}_{2}\right)_{\mathrm{x}} \mathrm{PPh}_{2}$ by $\mathrm{PPh}_{2}\left(\mathrm{CH}_{2}\right)_{\mathrm{x}} \mathrm{P}(\mathrm{O}) \mathrm{Ph}_{2}$ the bonding situation changes considerably due to phosphine $\pi$-acidity, the concerned orbitals being a mixture of $3 \mathrm{~d} \pi$ and P-C $\sigma^{*}$ components $^{12}$. There are thus two good $\pi$-acceptors in the phosphine complexes: dppz and $\mathrm{PPh}_{2}\left(\mathrm{CH}_{2}\right)_{\mathrm{x}} \mathrm{P}(\mathrm{O}) \mathrm{Ph}_{2}$. The back-bonding effect is maximized in the facial disposition which ensures minimum competition between the two ligands for identical metal orbitals. The observed geometrical selectivity is strong and exclusive. The strong geometrical discrimination by phosphine oxide and phosphine ligands is emerging as a general phenomenon ${ }^{4,6-9}$ for $\mathrm{ReCl}_{3}(\mathrm{NN})$ complexes where $\mathrm{NN}$ is a chelated $\pi$-acidic ligand. The present dipyridylpyridazine complexes augment this class. 
The voltammograms of $\mathbf{6}$ (initial scan cathodic) are virtually superimposable on those of the corresponding 2 species (initial scan anodic). The $t_{2 g}$ shell in $\mathbf{3}$ is stabilised by backbonding to phosphorus (vide infra) and an increase of the reduction potential is indeed expected in going from phosphine oxide to phosphine coordination ${ }^{5,8}$.

Oxygen atom transfer

The rate of the reaction of Eq. 2 has been determined spectrophotometrically in dichloromethane solution at $308 \mathrm{~K}$ for the $\mathrm{x}=4$ case where the subsequent isomerization proceeds very slowly. Time evolution spectra characterized by isosbestic point is shown in Figure 2. In the presence of excess diphosphine (under pseudo-first order condition), the rate $\left(k_{\text {oat }}\right)$ of transfer is proportional to the concentration of $\mathbf{1}$ and the observed rate constant, $k_{\text {obsd, }}$, is proportional to the concentration of phosphine implying a second order rate law (Eq. 7). Rate data are listed in Table 1.

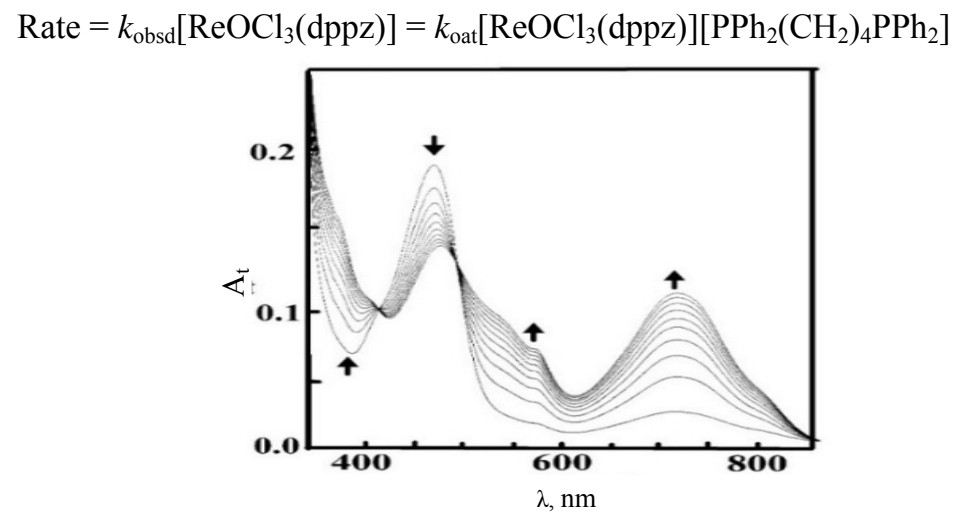

Figure 2. Time evolution spectra for the reaction of $\mathrm{ReOCl}_{3}(\mathrm{dppz})$ with $\mathrm{PPh}_{2}\left(\mathrm{CH}_{2}\right)_{4} \mathrm{PPh}_{2}$ in $\mathrm{CH}_{2} \mathrm{Cl}_{2}$ solution at $308 \mathrm{~K}$

Oxygen atom transfer to the both ends of $\mathrm{PPh}_{2}\left(\mathrm{CH}_{2}\right)_{\mathrm{x}} \mathrm{PPh}_{2}$ has been achieved by reacting the phosphine oxide complexes 2 (having dangling phosphine function) with excess $\mathrm{ReOCl}_{3}(\mathrm{dppz}), \mathbf{1}$ in dichloromethane. In the $\mathrm{x}=1$ case only the mononuclear product, 4 (Eq. 4) incorporating a dangling phosphine oxide function could be isolated. No binuclear product was observable. The reaction of $2(x=2-4)$ with excess 1 , in dichloromethane solution furnished the binuclear complex 5, Eq. 5 insoluble in all common organic solvents.

Table 1. Rate constants for the for the reaction of $\left[\mathrm{ReOCl}_{3}(\mathrm{dppz})\right]+\mathrm{PPh}_{2}\left(\mathrm{CH}_{2}\right)_{4} \mathrm{PPh}_{2} \rightarrow$ $\left[\mathrm{Re}\left(\mathrm{OPPh}_{2}\left(\mathrm{CH}_{2}\right)_{4} \mathrm{PPh}_{2}\right) \mathrm{Cl}_{3}(\mathrm{dppz})\right]$ in dichloromethane solution ${ }^{\mathrm{a}, \mathrm{b}}$.

\begin{tabular}{cccc}
\hline $\mathrm{T}, \mathrm{K}$ & $10^{2}\left[\mathrm{PPh}_{2}\left(\mathrm{CH}_{2}\right)_{4} \mathrm{PPh}_{2}\right], \mathrm{M}$ & $10^{4} \mathrm{k}_{\text {obsd }}, \mathrm{s}^{-1}$ & $10^{2} \mathrm{k}_{\text {oat }}, \mathrm{M}^{-1} \mathrm{~s}^{-1}$ \\
\hline \multirow{3}{*}{308} & 1.06 & 5.97 & \\
& 1.40 & 8.04 & $5.96(0.04)$ \\
& 2.00 & 11.60 & \\
\hline
\end{tabular}

${ }^{a}$ The initial concentration of $\left[\mathrm{ReOCl}_{3}(\mathrm{dppz})\right]$ is $1.25 \times 10^{-4} \mathrm{M} .{ }^{b}$ Least-squares deviations are given in parentheses

\section{Twin isomerization}

The spontaneous twin isomerization reaction of Eq. 3 was studied spectrophotometrically in dichloromethane solution for $\mathrm{x}=1-4$. A representative time evolution spectra is shown in Figure 3. The reaction followed a first-order rate law Eq. 8 implying that the reaction is 
intramolecular. Rate data are listed in Table 2. The variable temperature measurement of rate constants for $\mathrm{x}=1$ complex followed Eyring equation Eq. 9 and the activation parameters $\Delta \mathrm{H}^{\#}$ and $\Delta \mathrm{S}^{\#}$ are 15.18(0.02) kcal mol ${ }^{-1}$ and $-27.70(0.08) \mathrm{cal} \mathrm{K}^{-1} \mathrm{~mol}^{-1}$.

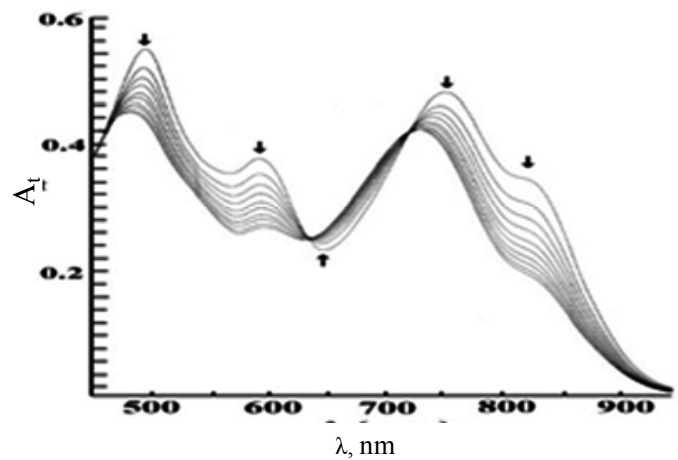

Figure 3. Time evolution spectra for the twin isomerization reaction of $2(x=1)$ in dichloromethane solution at $308 \mathrm{~K}$ ( $A_{\mathrm{t}}$ is absorbance)

Table 2. Rate constants for the reaction $\mathbf{2} \rightarrow \mathbf{3}$ in dichloromethane solution ${ }^{\mathrm{a}}$

\begin{tabular}{cccl}
\hline $\mathrm{x}$ & $\mathrm{T}, \mathrm{K}$ & $10^{4}[2], \mathrm{M}$ & $10^{5} \mathrm{k}_{\mathrm{ti}}\left(\mathrm{s}^{-1}\right)$ \\
\hline 1 & 308 & 1.25 & $12.20(0.03)$ \\
1 & 308 & 2.50 & $12.27(0.04)$ \\
1 & 308 & 3.75 & $12.15(0.03)$ \\
1 & 305 & 1.25 & $9.47(0.02)$ \\
1 & 302 & 1.25 & $7.33(0.03)$ \\
2 & 308 & 1.25 & $5.98(0.02)$ \\
3 & 308 & 1.25 & $3.21(0.02)$ \\
4 & 308 & 1.25 & $0.77(0.02)$ \\
\hline${ }^{a}$ Least-squares deviations are given in parentheses \\
Rate $=k_{\mathrm{ti}}\left[\operatorname{Re}\left(\mathrm{OPPh}_{2}\left(\mathrm{CH}_{2}\right)_{\mathrm{x}} \mathrm{PPh}_{2}\right) \mathrm{Cl}_{3}(\right.$ dppz $\left.)\right]$ \\
$k_{\mathrm{ti}}=\left(k_{\mathrm{B}} T / h\right)\left[\exp \left(-\Delta H^{\#} / R T\right) \exp \left(\Delta S^{\#} / R\right]\right.$
\end{tabular}

It is logical to assume that the transformation is initiated via nucleophilic attack of the metal by the dangling phosphorus atom. The attack is stylized in 7 . The transformation can then proceed via edge displacement ${ }^{7-9}$ of a chloride ligand resulting in relay substitutions: $\mathrm{Re}-\mathrm{OP}$ by $\mathrm{Re}-\mathrm{Cl}$ and of $\mathrm{Re}-\mathrm{Cl}$ by $\mathrm{Re}-\mathrm{P}$. The net effect is twin isomerization (7). An alternative pathway involving direct transfer of oxygen from phosphorus to phosphorus is energetically highly unlikely since it requires the synchronous dissociation of both $\mathrm{P}-\mathrm{O}$ and $\mathrm{Re}-\mathrm{O}$ bonds. The process is depicted in 7 evidently involves considerable ordering consistent with the large negative entropy of activation $\left(-27.70 \mathrm{cal} \mathrm{K}^{-1} \mathrm{~mole}^{-1}\right)$.

The rate of the twin isomerization reaction decreases rapidly as the spacer length increases from $x=1$ to $x=4$. Indeed the dependence of rate on $x$ is exponential to a good degree as illustrated in the plots of Figure 4. The data fits approximately with the relation given in Eq. 10 where $\mathrm{A}_{0}$ is a constant characteristic of the dppz ligand system. The values of the constants derived from the fits are $\mathrm{A}_{0}=0.0002450$ and $\mathrm{a}=1.7732$. The number of possible conformations of the dangling $\left(\mathrm{CH}_{2}\right)_{\mathrm{X}} \mathrm{PPh}_{2}$ fragment is indeed expected to increase exponentially as $\mathrm{x}$ increases ${ }^{8,9,13}$ but only a few of the conformations will be spatially suited (proximal metal and phosphine sites) for the reaction to occur. 


$$
k_{\mathrm{ti}}=\mathrm{A}_{0} \mathrm{e}^{-\mathrm{ax}}
$$

Upon oxidation of the metal to the tetravalent state as in $\mathbf{6}$, the isomerization process is completely arrested even when $x=1$ which corresponds to maximum reactivity in the case of 2.Coulometric reduction of $\mathbf{6}$ to $\mathbf{2}$ reestablishes the isomerization process. The lack of reactivity of 6 could be a reflection of the kinetic inertness of the $\mathrm{d}^{3}$ configuration in rhenium(IV). Other factors are also expected to work in the same direction. Thus rhenium(IV) is a harder acceptor than rhenium(III) and oxygen is a harder donor than phosphorus. This as well as the weaker back-bonding ability of rhenium(IV) is expected to disfavour the attack of the metal by the dangling phosphine function.

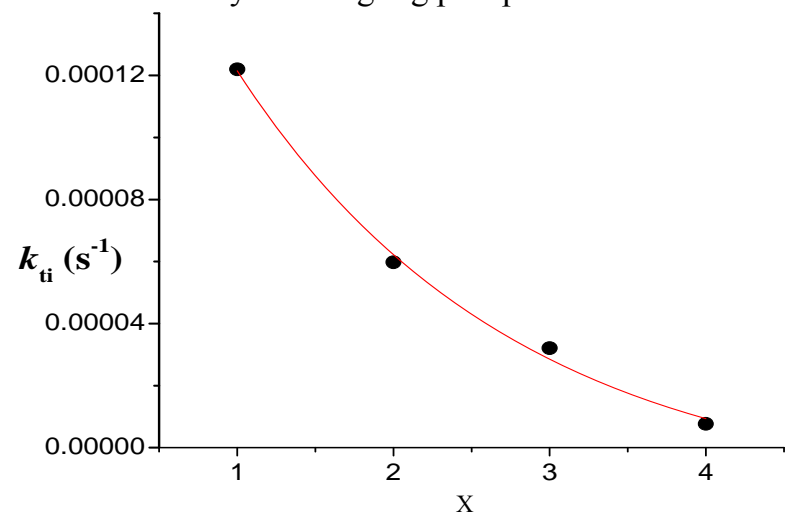

Figure 4. Exponential plot of rate constant versus diphosphine spacer length for the isomerization reaction of $\mathrm{Re}\left(\mathrm{OPPh}_{2}\left(\mathrm{CH}_{2}\right)_{\mathrm{x}} \mathrm{PPh}_{2}\right) \mathrm{Cl}_{3}(\mathrm{dppz}),(\mathrm{x}=1-4)$ in dichloromethane solution at $308 \mathrm{~K}$

\section{Conclusion}

It is demonstrated that the family $\mathrm{Re}^{\mathrm{III}}\left(\mathrm{OPPh}_{2}\left(\mathrm{CH}_{2}\right)_{\mathrm{x}} \mathrm{PPh}_{2}\right) \mathrm{Cl}_{3}(\mathrm{dppz}), \mathbf{2}$ with dangling phosphine function formed from $\mathrm{Re}^{\mathrm{V}} \mathrm{OCl}_{3}(\mathrm{dppz}), \mathbf{1}$ and $\mathrm{PPh}_{2}\left(\mathrm{CH}_{2}\right)_{\mathrm{x}} \mathrm{PPh}_{2}$ (where $\mathrm{x}=1-4$ ) undergoes spontaneous intramolecular linkage-cum-geometrical isomerization in solution affording $\mathrm{Re}^{\mathrm{III}}\left(\mathrm{PPh}_{2}\left(\mathrm{CH}_{2}\right)_{\mathrm{x}} \mathrm{P}(\mathrm{O}) \mathrm{Ph}_{2}\right) \mathrm{Cl}_{3}(\mathrm{dppz})$, 3. The complex $2(\mathrm{x}=1)$ upon reaction with excess 1 affords mononuclear $\mathrm{Re}^{\mathrm{III}}\left(\mathrm{OPPh}_{2}\left(\mathrm{CH}_{2}\right) \mathrm{P}(\mathrm{O}) \mathrm{Ph}_{2}\right) \mathrm{Cl}_{3}(\mathrm{dppz}), 4$ while for $\mathrm{x}=2-4$ binuclear complex $(\mathrm{dppz}) \mathrm{Cl}_{3} \mathrm{Re}^{\mathrm{III}}\left(\mathrm{OPPh}_{2}\left(\mathrm{CH}_{2}\right)_{\mathrm{x}} \mathrm{PPh}_{2} \mathrm{O}\right) \mathrm{Re}^{\mathrm{III}} \mathrm{Cl}_{3}(\mathrm{dppz}), \mathbf{5}$ is obtained. Significant $\mathrm{Re}^{\mathrm{III}}(\mathrm{dppz})(2-4)$ and $\mathrm{Re}^{\mathrm{III}} \mathrm{P}$ (3) back-bonding characterizes the species and this logically plays a crucial role in directing the course of isomerization and in determining the trend $(3>2,4)$ of MLCT excitation energy and $\mathrm{Re}^{\mathrm{IV}} / \mathrm{Re}^{\mathrm{III}}$ reduction potential. The twin isomerization process $\mathbf{2} \rightarrow \mathbf{3}$ is unimolecular and is associated with considerable ordering in the transition state (large negative entropy of activation) and a reaction pathway has been proposed. The rate of twin isomerization reaction falls rapidly with increasing $\mathrm{x}$ as expected from the statistics of polymethylene conformation. The isomerization halts completely upon metal oxidation even for $\mathrm{x}=1$ species believed to be due to kinetic inertness of $\mathrm{d}^{3}$ configuration and weaker back-bonding ability of Re(IV).

\section{References}

1. Volkert W A and Jurisson S, Top Curr Chem., 1996, 176, 123-148.

2. Dilworth J R and Parrott S J, Chem Soc Rev., 1998, 27, 43-55, DOI:10.1039/A827043Z; (b) Hashimoto K and Yoshihara K, Top Curr Chem., 1996, 
176, 275-291; (c) Volkert W A and Hoffman T J, Chem Rev., 1999, 99, 2269-2292, DOI:10.1021/cr9804386 (d) Espenson J H, Adv Inorg Chem., 2003, 54, 157-202; DOI:10.1016/S0898-8838(03)54003-X (e) Espenson J H, Coord Chem Rev., 2005, 249, 329-341, DOI:10.1016/j.ccr.2004.06.018

3. Couillens X, Gressier M, Dartiguenava M, Fortin S and Beauchamp A L, J Chem Soc, Dalton Trans., 2002, 3032-3036, DOI:10.1039/B201632J (b) Bereauchamp A L, Khan S I and Abu-Omar M M, Inorg Chem., 2001, 40, 6767-6773; DOI:10.1021/ic0108033

4. Chakravorty A, Eur J Inorg Chem., 2005, 4863-4874, DOI:10.1002/ejic.200500615 (b) Abram U, Comprehensive Coordination Chemistry II, Volume 5, Edited by McCleverty J A and Meyer T J, 2004, Elsevier, Oxford.

5. Sengupta S and Chakravorty A, J Ind Chem Soc., 2006, 83, 556-562; (b) Gangopadhyay J, Sengupta S, Bhattacharyya S, Chakraborty S and Chakravorty A, Inorg Chem., 2002, 41, 2616-2622, DOI:10.1021/ic011064t; (c) Seymore S B and Brown S N, Inorg Chem., 2000, 39, 325-332, DOI:10.1021/ic990851b; (d) Bryan J C, Stenkamp R E, Tulip T H and Mayer J M, Inorg Chem., 1987, 26(14), 2283-2288, DOI:10.1021/ic00261a024; (e) Rowbottom J F and Wilkinson G, J Chem Soc, Dalton Trans., 1972, 826-830, DOI:10.1039/DT9720000826; (f) Hille R, Chem Rev., 1996, 96(7), 2757-2816, DOI:10.1021/cr950061t; (g) Holm R H, Kennepohl P and Solomon E I, Chem Rev., 1996, 96(7), 2239-2314, DOI:10.1021/cr9500390

6. Panda B K, Senapati U, Mondal B and Sengupta S, Chem Sci Trans., 2015, 4(2), 337-346; DOI:10.7598/cst2015.998

7. Bhattacharyya S, Chakraborty I, Dirghangi B K and Chakravorty A, Chem Commun., 2000, 1813-1814, DOI:10.1039/B004565I; (b) Bhattacharyya S, Chakraborty I, Dirghangi B K and Chakravorty A, Inorg Chem., 2001, 40, 286-293; DOI:10.1021/ic0005893

8. Sengupta S and Chakravorty A, J Ind Chem Soc., 2006, 83, 961-972.

9. Chakraborty I, Sengupta S, Das S, Banerjee S and Chakravorty A, Dalton Trans., 2003, 134-140, DOI:10.1039/B208338H; Sengupta S, Gangopadhyay J and Chakravorty A, Dalton Trans., 2003, 4635-4643, DOI:10.1039/B307834E

10. Nakamoto K, Infrared and Raman Spectra of Inorganic and Coordination Compounds, Theory and Applications in Inorganic Chemistry, Part A, 1997, John Wiley and Sons, Inc., New York, $5^{\text {th }}$ Edn., pg 182.

11. Chakraborty I, Bhattacharyya S, Banerjee S, Dirghangi B K and Chakravorty A, $J$ Chem Soc, Dalton Trans., 1999, 3747-3753, DOI:10.1039/A906460E

12. Cotton F A and Wilkinson, G, Advanced Inorganic Chemistry, 1988, Wiley, New York, $5^{\text {th }}$ Edn., 64.

13. Flory P J, Principles of Polymer Chemistry, 1990, Cornell University Press, Ithaca and London, $4^{\text {th }}$ Edn., 399. 